\title{
A novel opto-mechanical system constituted by LEDs to employment in photobiostimulation: Clinical application in optical therapy
}

\author{
Celso J. Barbosa ${ }^{\mathrm{a}}$, Crisaldo Teles ${ }^{\mathrm{a}}$, Leonardo M. Moreira ${ }^{\mathrm{b}, *}$, Álvaro J. Damião ${ }^{\mathrm{c}}$ and \\ Carlos J. de Lima ${ }^{\text {a }}$ \\ ${ }^{a}$ Laboratório de Instrumentação Biomédica, Centro de Engenharia Biomédica, Universidade Camilo \\ Castelo Branco, Parque Tecnológico de São José dos Campos, São José dos Campos, SP, Brazil \\ ${ }^{\mathrm{b}}$ Universidade Federal de São João Del Rei, São João Del Rei, MG, Brazil \\ ${ }^{\mathrm{c}}$ Laboratório de Medição de Superfícies Ópticas-LMSO-IEAv-CTA, São José dos Campos, São Paulo, \\ SP, Brazil
}

\begin{abstract}
Optical techniques of photobiostimulation, which use transducer as lasers and LEDs, have been employed in the treatment of several diseases. The laser systems usually irradiate in a reduced area of the target biological tissue, presenting high cost of acquisition. Devices with a LED of "Surface Mount Device" (SMD) type have high angle of light emission, implying in intense decrease of the density of optical potency. Furthermore, the use of this device requires the utilization of a cooling system, which provokes an increase in the cost of this product. In the present work, it is suggested the application of a novel opto-mechanical device constituted by usual LEDs with package dimension of $5 \mathrm{~mm}$ of diameter, which presents significant efficient and very low cost. The arrangement of the geometric configuration of these LEDs is elaborated in such way that all units emit photons in a unique section of an specific area. An opto-mechanical system was developed, which includes seven LEDs, being that six of these LEDs are disposed in a specific angle around a unique central LED. It is important to notice that all the opto-mechanical system with seven LEDs emit light in a unique area. Besides, a methodology was employed to allow the verification of the distribution of light intensity upon this respective area. This arrangement allows that this novel device propitiate measurements of the degree of homogeneity of the light intensity in specific areas, which are accessed by the seven light emitting units. The potency employing LEDs in the red (visible) and near infrared regions were also measured, being that the result demonstrated the capability of clinical application in optical therapy of photobiostimulation, which was also used in a pilot test of clinical application.
\end{abstract}

Keywords: LEDs, photobiostimulation, opto-mechanical system

\section{Introduction}

Several works that are focused on the photobiostimulation technique applied to clinical therapy employ a transducer of light, such as Laser and Light Emitting Diodes (LEDs) [12,13,21,22]. These studies

\footnotetext{
${ }^{*}$ Corresponding author: Leonardo M. Moreira, Universidade Federal de São João Del Rei, São João Del Rei, Minas Gerais, Brazil. E-mail: leonardomarmo@gmail.com.
} 
demonstrated that photobiostimulation is an excellent coadjuvant technique to the treatment of various diseases. In fact, photobiostimulation has been employed as supporting therapy for the treatment of a great number of lesions. These works demonstrated that the light incidence with specific spectral range and adequate optical intensity $\left(\mathrm{J} / \mathrm{cm}^{2}\right)$ on the biological tissue provokes an increase in ATP production [2,9-11], which generates a biological process that results in the pain quenching, cell proliferation increase, inflammation decrease, etc.

On the other hand, the optical system constituted by laser presents some limitations. Indeed, the light incidence reaches a very restrict area and, if this lesion processes a higher size, it would be necessary to apply the light in several positions around the biological target in order to obtain the clinical results in the respective treatment [3]. Moreover, it is important to notice that the laser systems present high cost, making the acquisition difficult for a great number of healthy professionals.

The LED systems that are available for the healthy units present a very lower cost, and have a much more suitable area of light incidence when compared with lasers. This interesting property allows to use the light incidence in a unique application. The LED equipment available for clinical utilization has a package configuration of Surface Mount Device (SMD) type, which presents an appropriate optical potency of approximately hundreds of milliwatts. On the other hand, the light emission angle has a very high value (around $120^{\circ}$ ) [14], being that this angle furnishes a very extensive irradiation area, which implies in a decrease of the potency density in $\mathrm{mW} / \mathrm{cm}^{2}$ in order to attenuate a significant variation of optical power density. This same type of LED generates a consumption of significant percentage of electric potency in thermal energy. In this way, it is necessary that this kind of device eliminates this thermal energy through a heat-sinking substrate, being necessary the employment of an air refrigeration system, that is, the cooling with a fan. These considerations results in an increase in the cost of this LED system of illumination.

Usually, it is employed a common LED with capsule constituted with transparent resin (Fig. 1), which presents a very low cost in relation to the SMD LED. In fact, this common LED costs less than one dollar. In the present work, it is proposed the development of an electronic-opto-mechanical device that uses a simple package LED as demonstrated in Fig. 1, with a geometric arrangement in which all LEDs emit light in a unique area, resulting in an intensity of total light with a value suitable to application in photobiostimulation or phototherapy. In this work, the application of this new prototype is presented, including some optoelectronics measurements performed in laboratory in order to evaluate the potential of this new equipment in application in biomedical engineering.

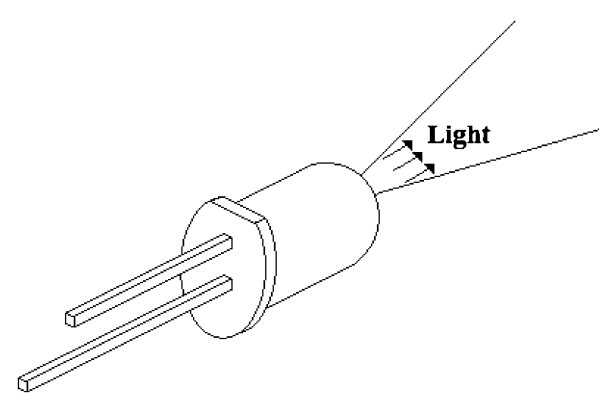

Fig. 1. Conventional LED package configuration frequently available to acquisition, which presents package dimension with external diameter of $5 \mathrm{~mm}$ and frontal light emission. 


\section{The novel device}

The new device was projected in order to employ seven LEDs in a unique optical system, in agreement with Fig. 1, in a such way that the final geometric configuration is constituted by six LEDs disposed around a unique central LED [5-8]. The specific inclination angle of the six LEDs that involve the central LED is adequate to an optimum light incidence on the biological tissue, considering the approximated distance between the light source and the biological target. In this way, the total intensity of incident light would be the summation of the light intensity of seven LEDs (Fig. 2(a)-(c)). The design presented in Fig. 2(c), demonstrates the disposition of each light emitter with a specific angle $(\theta)$, being that this value of inclination is determined as function of two factors: the first factor is the measurement of the distance between the LEDs, which is indicating by distance B, in Fig. 2(c). This value is determined considering the diameter of the LED package of the light emitter, adding with a space between the LEDs to applications in mechanical structure (usually $1 \mathrm{~mm}$ ). The second measurement corresponds to the height A as demonstrated in Fig. 2(c), being that this distance is called by "working distance". This value is considered as function of the divergence angle of the light from LED (Fig. 1), and the diameter that determine the target area ("hotspot") to be irradiated (tissue). These light emitters must be electrically polarized in such way that the stability of the light intensity is maintained. In this way, on the area to be irradiated would be a resulting homogeneity in terms of light intensity distribution, with suitable optical power value to application in photobiostimulation. In this way, it is necessary the utilization of a technique to verification of this distribution of light intensity in all areas to be considered.

\section{Materials and methods}

Seven common LEDs (SuperBright LEDs) of package dimension of $5 \mathrm{~mm}$ of diameter, which emit light with $627 \mathrm{~nm}$ of wavelength $(\lambda=627 \mathrm{~nm})$, and part number RL5-R12008 [19] were employed and

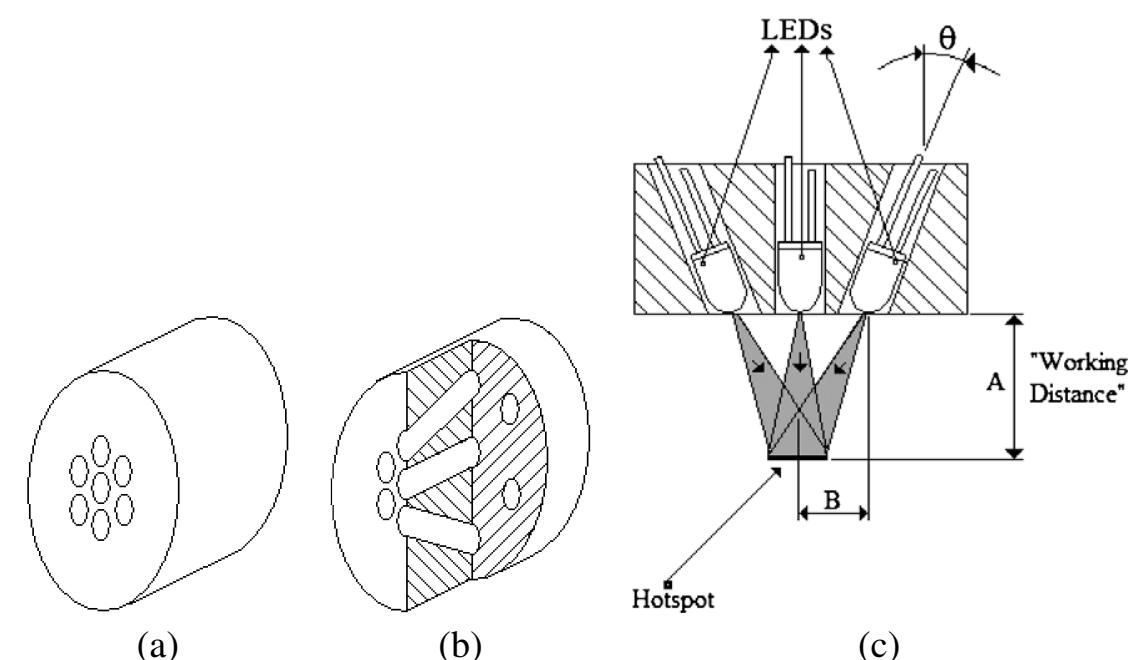

Fig. 2. Simplified scheme of the geometric configuration of the arrangement of the seven LEDs of package dimension of $5 \mathrm{~mm}$ of diameter: (a) demonstrates the tridimensional design of the device of the arrangement of LEDs; (b) illustrates the inclination of the light emitters around the central LED; (c) shows that, at the "working distance", the photons of the light emitters are incident in a unique area ("hotspot"). 
arranged in a unique equipment as demonstrated in Fig. 2. These monochromatic light emitters were electrically polarized, using an electronic circuit that allows the stability of the electric current that flows in the LEDs, precluding the variation of the light intensities. This same electronic circuit system was dimensioned in such way that the light emitters act in the limits of the parameters furnished by the data sheet of the LED. A second opto-mechanical device was elaborated utilizing the same type of package, which presents $5 \mathrm{~mm}$ of diameter. These LEDs were purchased from the same company, as mentioned previously. The wavelength emitted by this new device is $945 \mathrm{~nm}(\lambda=945 \mathrm{~nm})$, being that its part number is RL5-IR2730 [20]. These equipments was elaborated with the electronics circuits adequate to the properties of the respective component, resulting in the constitution of two devices (one of them with LEDs acting in the visible spectral region and other in the near infrared region). To perform the measurements of optical power of the two systems mentioned, the instrument ThorLab model PM100D with calibration certificate dully updated was employed.

In order to verify the distribution of light intensity in the target area, an experiment that employed an apparatus to measure this homogeneity was performed. For this aim, the experiment utilized a support that allows the shift (in micrometers) in the two orthogonal directions $X Y$. In this same support, that permits the shift in both orthogonal directions $X Y$ (Fig. 3), it was fixed an equipment, in which presented a hole miniature of $0.300 \mathrm{~mm}$ diameter. In a proximal position of this orifice, an optoelectronic component that acts as photo-sensor with miniaturized hole was positioned. This component is known as "photodiode". The photodiode is electrically polarized and connected to a voltmeter (electronic meter). Above this assemblage with the photodiode, it was fixed the system with seven LEDs proposed and, in this way, from different distances between the photo-sensor and the respective device, it is possible to

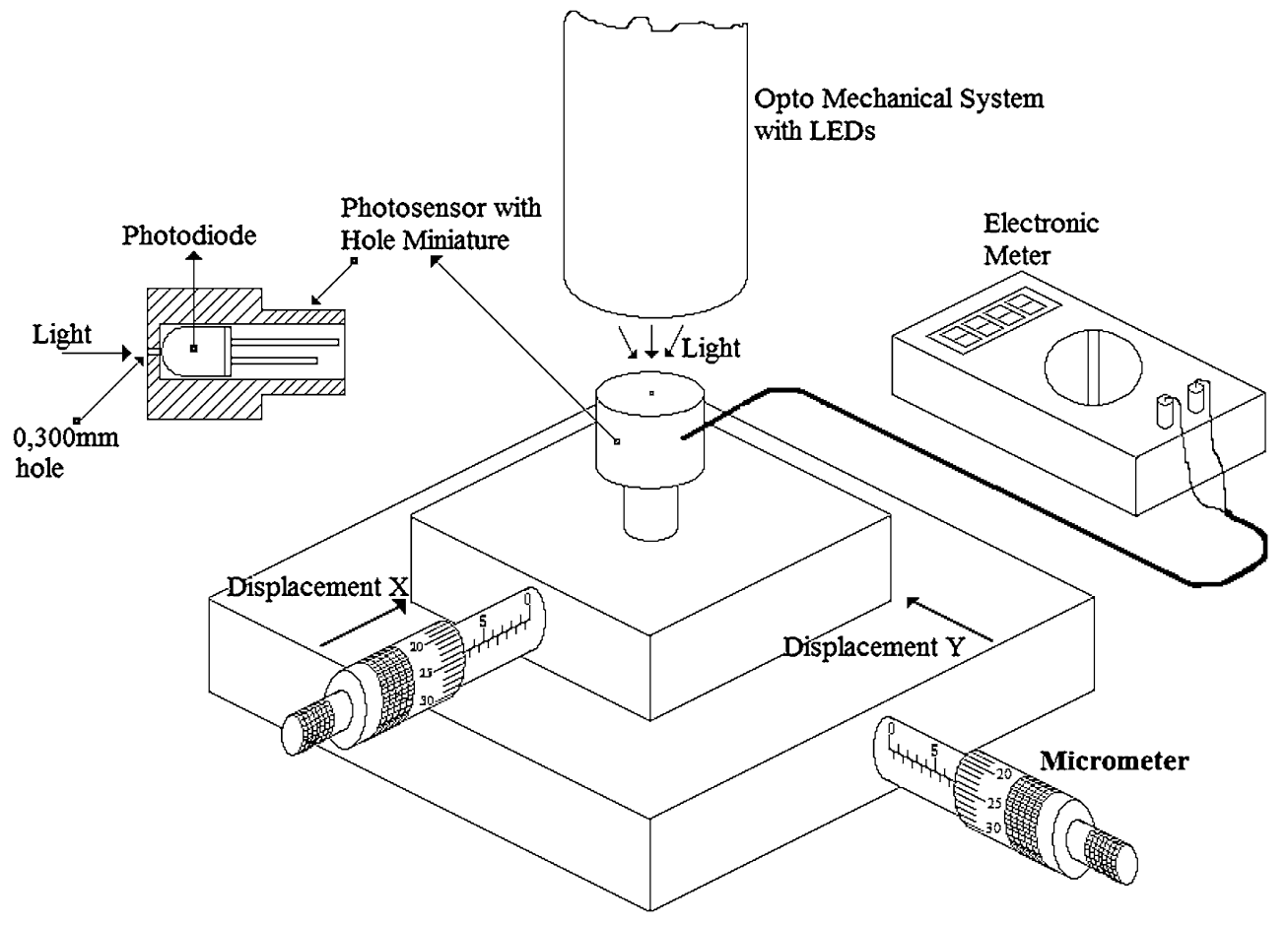

Fig. 3. Experimental apparatus for obtaining the measurement of light intensity distribution of the LEDs system. 
measure indirectly the light intensity via voltmeter. Considering that the light is emitted by a very small area $(0.300 \mathrm{~mm}$ of diameter), a discrete optical measurement is available to each value. In this way, the distance of $X$ and/or $Y$ can be modified in $0.500 \mathrm{~mm}$. In this way, a table is obtained through a software (Microsoft Excel), and it was possible to delineate the curves on the surfaces.

\section{Results}

The measurements of optical intensity, employing the ThorLab instrument were developed in agreement with previous description. This measurement was accomplished with light of wavelength $627 \mathrm{~nm}$ $(\lambda=627 \mathrm{~nm})$ and showed a potency of $70 \mathrm{~mW}$. On the other hand, another optical system with seven LEDs that irradiates light in the near infrared spectral range $(\lambda=945 \mathrm{~nm})$, propitiated the value of $135 \mathrm{~mW}$.

According to the experimental apparatus represented in Fig. 3, it was possible to obtain the graphics of light intensity distribution of the LEDs system elaborated in the present work. In fact, with the analysis of the intensities obtained step by step, following the shift in micrometers (to a distance of $15 \mathrm{~mm}$ from photo-sensor until the seven LEDs), the methodology was able to elaborate a table of 28 lines and 32 columns, resulting in a total number of 896 measuring points, being that each value represents a specific intensity. To this elaboration, it is employed a graphic software (Microsoft Excel), and a curve of surface type was plotted, in agreement with Fig. 4.

Figure 4 demonstrates the presence of peaks, which presents bases with diameter ranges between 10.5 and $12 \mathrm{~mm}$. In this graphic, the distance between the photo-sensor with miniaturized hole and the optical system (with seven LEDs) was $15 \mathrm{~mm}$. Another measurement employed the same arrangement with a small difference. In this second case, the measurement adjusted between the photo-sensor and the optical

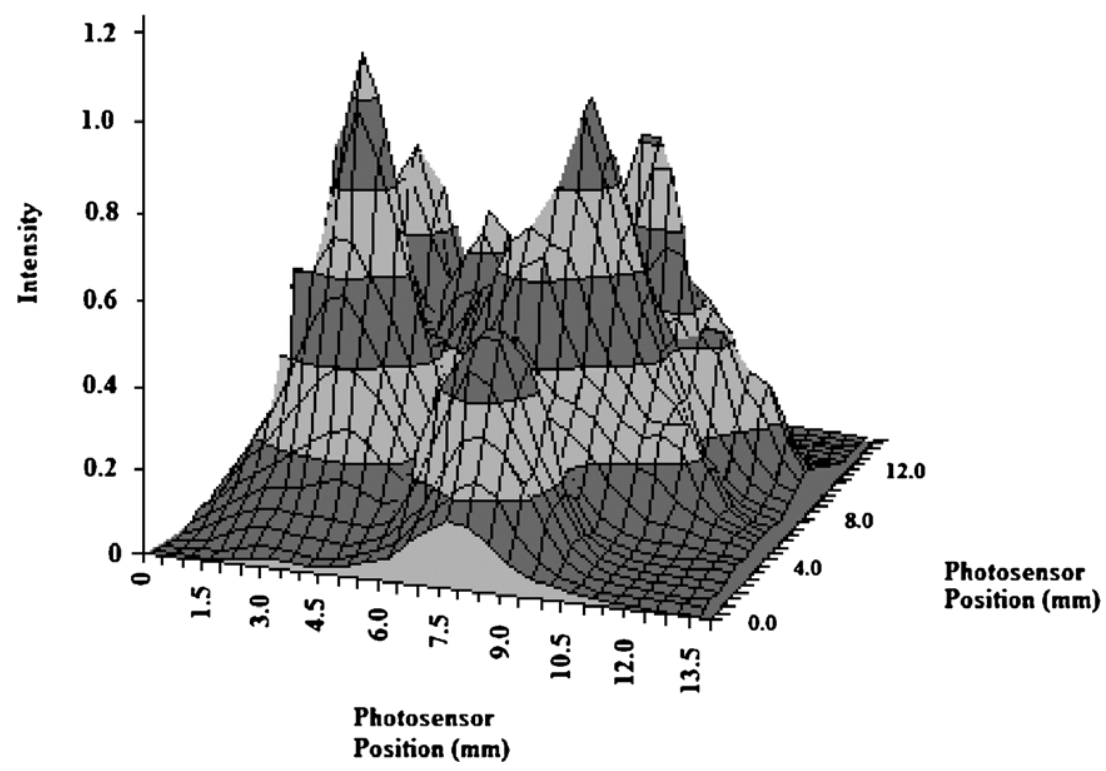

Fig. 4. Graphic of the surface type indicating the distribution of the light intensity of the optical system with seven LEDs, being that the distance between the novel device and the photodiode was $15 \mathrm{~mm}$. 


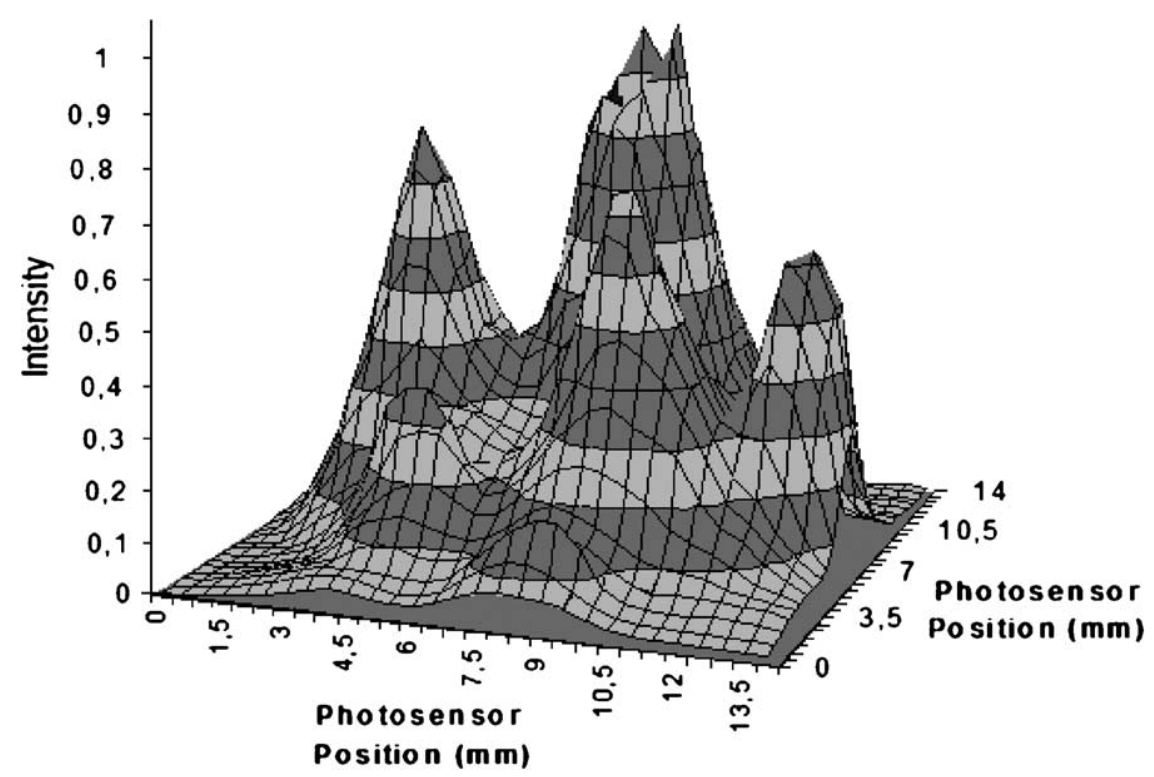

Fig. 5. Graphic of surface type indicating the light intensity distribution of the optical system with seven LEDs, the distance between the novel device and the photodiode was $20 \mathrm{~mm}$.

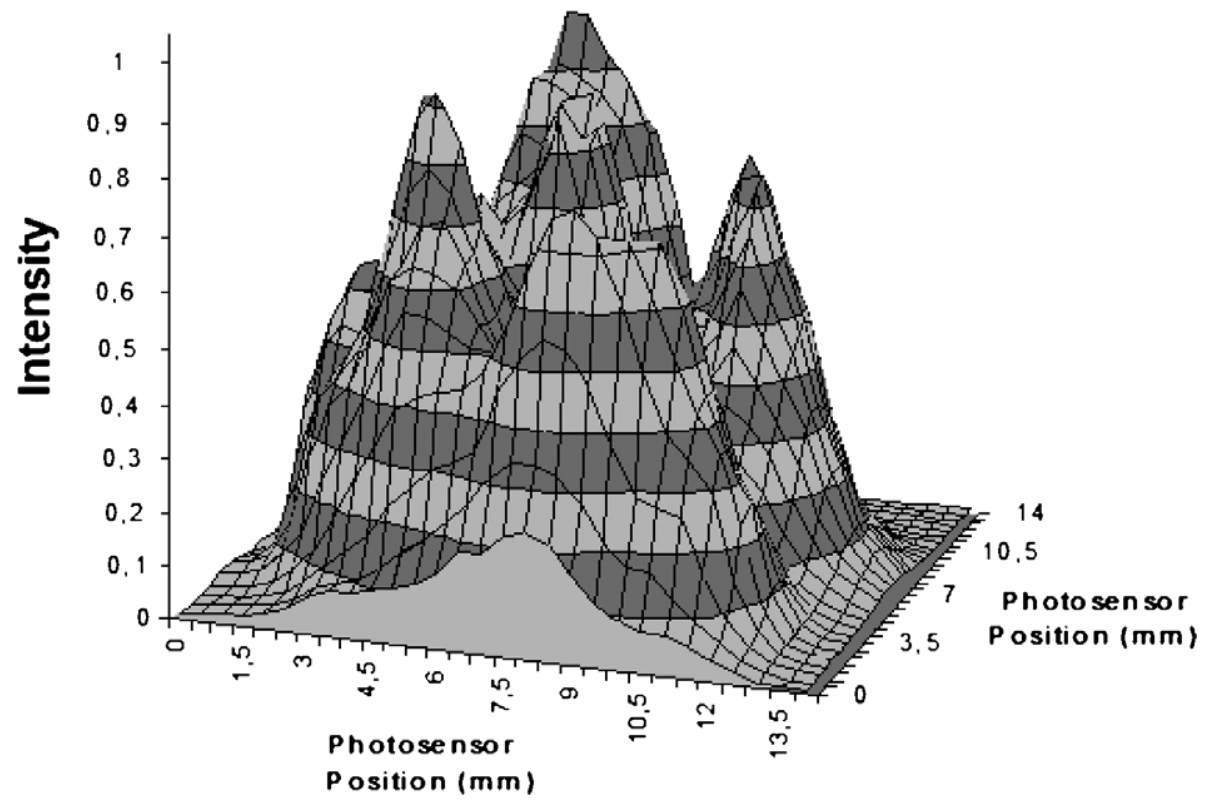

Fig. 6. Graphic of the surface type indicating the light intensity distribution of the novel optical system with seven LEDs, the distance between the novel device and the photodiode was $25 \mathrm{~mm}$.

system of seven LEDs was $20 \mathrm{~mm}$. Figure 5 also demonstrates the presence of peaks of light intensity in which the respective bases have diameters between 10.5 and $12 \mathrm{~mm}$.

Figure 6 also indicates the light intensity distribution of the novel optical system with seven LEDs, being that the distance between the photo-sensor and the arrangement of light emitter was $25 \mathrm{~mm}$, as it 
can be observed in the three graphics previously mentioned. Figure 6 demonstrates the presence of three peaks of light intensity, which suggests that each peak represents the light emission of each specific LED.

\section{Discussion}

In this work, the development of an optical system constituted by seven common LEDs of package dimension of $5 \mathrm{~mm}$ of diameter is proposed. These optoelectronic components present low cost and does not generated thermal dissipation. Furthermore, this novel equipment also present a small range of angles $\left(8^{\circ}-30^{\circ}\right)$. In the case of the phototherapic equipment constituted by a LED of the SMD type, in spite of the emission of high optical potency (hundreds of milliwatts), this equipment presents a divergence angle very significant (from $90^{\circ}$ until $140^{\circ}$ ). In this way, the variation of the distance between the target and the light emitter provokes a significant difference in the irradiated area, which results in an important modification of the optical power density. Furthermore, the LED of high power remains stable in its emission and requires a cooling system ("heat dissipation"), which is constituted by a metallic substrate ("heat sink") and a small fan to electric-thermical stability of the respective component. All details result in a high cost equipment, while the novel optical system presently suggested is much more accessible. In this work, it is proposed a new optical system constituted by LEDs with common package dimension of $5 \mathrm{~mm}$ diameter, in which it is utilized an arrangement with seven LEDs with six of them around one central LED, allowing that, in a specific distance of the light emitters until the target, all these LED units emit light in a unique area, which is a very interesting property in order to be applied in processes involving photobiostimulation. In fact, the novel devices emit light with wavelength of 627 and $945 \mathrm{~nm}$, respectively, presenting optical power of 70 and $135 \mathrm{~mW}$. These values are suitable to application in photobiostimulation, which can be inferred in previous works that presented the employment of these devices emitting in the red region (approximately $630 \mathrm{~nm}$ ) $[1,15,16]$.

The two kinds of devices were elaborated in such a way that the called "working distance" as well as the angle $\theta$ generated in a determined space (associated to a diameter of $1.13 \mathrm{~cm}$ ) were very adequate to the application in photobiostimulation. Indeed, these conditions result in an area of $1 \mathrm{~cm}^{2}$, which means that the optical power measured from the optical instrument (optical power meter), already indicates the own value of optical density. Really, observing the graphics of light intensity distribution of LEDs, there is evidence of a range of diameters between 1.05 and $1.2 \mathrm{~cm}$, which demonstrates compatibility with the specified nominal value.

Therefore, this work present a novel optical device of very low cost and high performance proposing its employment in photobiostimulation processes. To the efficiency of this kind of applications is necessary the specification of the distance between the target biological tissue and the arrangement of light emitters. In order to obtain this optimum condition it is necessary the specification of the suitable distance between the target tissue and the optical apparatus, implying in the employment of a spacer. Indeed, in the tip of the new equipment that contains the LED units a spacer it was positioned aiming to maintain the light incidence in the specific diameter of interest in the respective therapeutic methodology, in agreement with previous discussion (Fig. 7). The target biological area to be irradiated by LEDs is approximately of $1 \mathrm{~cm}^{2}$, which is an interesting value in comparison with the utilization of a LASER with a diameter of light incidence of around $1 \mathrm{~mm}$. This relation area, which can be considered approximately 128 -fold, denotes the excellent perspectives of clinical application of this new optical prototype. In this way, the use of LEDs, when the lesion to be treated has a relatively high size, would require a very lower number of regions to be irradiated. This advantage permits a very higher quality of life to the patients, limiting the occurrence of negative side effects as function of the number of light applications. 


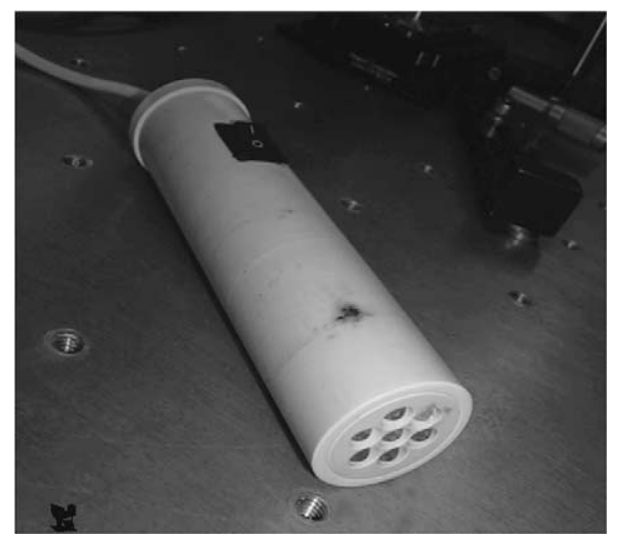

(a)

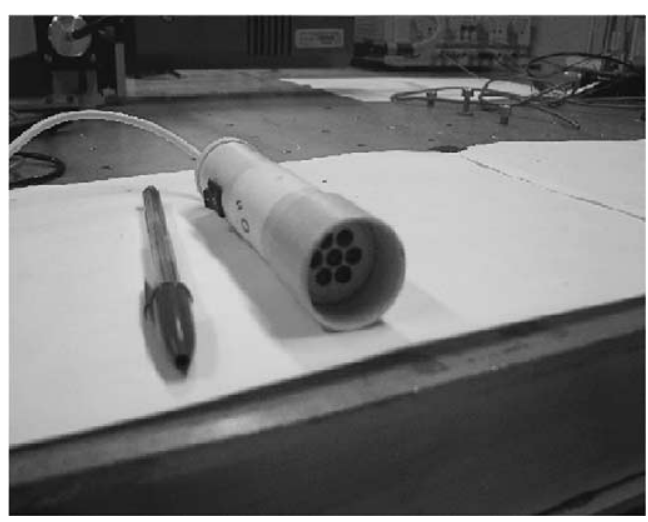

(b)

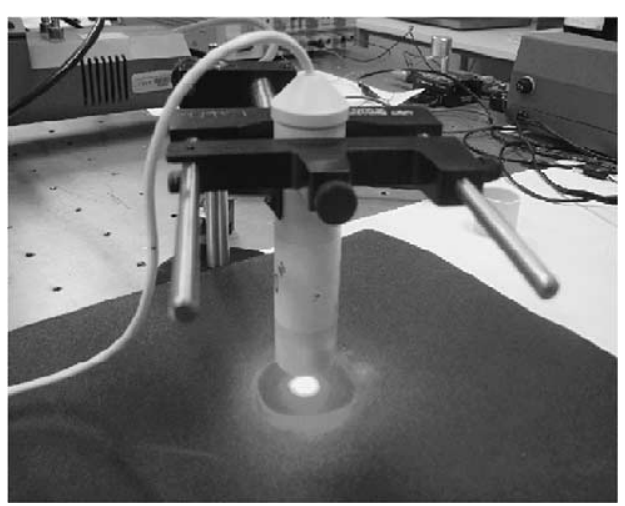

(c)

Fig. 7. Photos indicating the system of arrangement with common LEDs of five millimeters of diameter: (a) presents the image of the optical system with the LEDs configuration in the arrangement referent to the disposition of six LEDs around a central LED; (b) indicates the optical system constituted of LEDs with the specific apparatus to permit the suitable space between the light emitters and the biological target tissue; (c) shows a photography of the proposed optical system with LEDs focusing the light on a dark surface, which generate a bright area around $1 \mathrm{~cm}^{2}$ for a given "work distance".

The literature suggests that the photobiostimulation applied to several clinical cases, such as skin and musculoskeletal lesions, should act with an energy density value between 1 and $10 \mathrm{~J} / \mathrm{cm}^{2}[4,17,18]$, mainly in the dermatological application as local sensitivity, edema, ulcers, burns, etc. Considering the value of $70 \mathrm{~mW}$, which was measured in this novel prototype, the maximum time of light exposition to these LEDs would be of approximately three minutes, which is a very reasonable value of the clinical point of view.

To the clinical application in Photodynamic Therapy (PDT), in which the dose involves values of approximately $100 \mathrm{~J} / \mathrm{cm}^{2}$ [21], an interesting option would be the elaboration of a similar arrangement of LEDs, but with 12 additional LEDs around the 6 LEDs that are the external LEDs of the present optical prototype. In this arrangement, the fixation of these LEDs present a spherical form, allowing that all LEDs to irradiate the light upon the same area, which corresponds to the center of the sphere. In fact, with these 19 light emitters, the total optical power would be approximately $200 \mathrm{~mW}$, which would result in an exposition time of around 9 min. 


\section{Conclusions}

This work demonstrated the suitability of a novel optical device to photodynamic applications. In fact, the optical system of LEDs presented in this manuscript has demonstrated great potential in photobiostimulation, with auspicious perspectives to clinical applications in the treatment of various diseases, mainly in skin, bone and muscle lesions.

\section{Acknowledgement}

The authors thank Luiz Fernando Alves for his contribution in terms of technical advice with respect to LEDs and mechanical components.

\section{References}

[1] M.A.D. Agnol, R.A. Nicolau, C.J. De Lima and E. Munin, Comparative analysis of coherent light action (laser) versus non-coherent light (light-emitting diode) for tissue repair in diabetic rats, Lasers Med. Sci. 24 (2009), 909-916.

[2] A. Amat, J. Rigau, R.W. Waynant, I.K. Ilev and J.J. Anders, The electric field induced by light can explain cellular responses to electromagnetic energy: a hypothesis of mechanism, J. Photochem. Photobiol. 82 (2006), 152-160.

[3] H. Beckerman, R.A. de Bie, H.J. De Cuyper and R.A.B. Oostendorp, The efficacy of laser therapy for musculoskeletal and skin disorders: a criteria-based metaanalysis of randomized clinical trials, Phys. Ther. 7 (1992), 483-491.

[4] T.Y. Chung, P.V. Peplow and G.D. Baxter, Laser photobiostimulation of wound healing: defining a dose response for splinted wounds in diabetics, Lasers Surg. Med. 42 (2010), 656-664.

[5] C.J. De Lima, L.M. Moreira, J.P. Lyon, A.B. Villaverde and M.T.T. Pacheco, Catheters: instrumental advancements in biomedical applications of optical fibers, Lasers Med. Sci. 24 (2009), 621-626.

[6] C.J. De Lima, M.T.T. Pacheco, A.B. Villaverde, R.A. Zângaro, L.M. Moreira and A.J. Damião, Catheter with dielectric optical filter deposited upon the fiber optic end for Raman in vivo biospectroscopy applications, Spectroscopy 22 (2008), 459-466.

[7] C.J. De Lima, S. Sathaiah, L. Silveira, Jr., R.A. Zângaro and M.T.T. Pacheco, Development of catheters with low background signals for Raman spectroscopy diagnosis applications, Artificial Organs 23 (2000), 231-234.

[8] C.J. De Lima, M. Simões, M.T.T. Pacheco, L. Silveira, Jr. and A.B. Villaverde, Optical fiber catheter with distal end bending mechanism control for Raman biospectroscopy, Instr. Sci. Technol. 26 (2008), 1-13.

[9] G. Drochioiu, Laser-induced ATP formation: mechanism and consequences, Photomed. Laser Surg. 28 (2010), $573-573$.

[10] M. Heger, A.A.M. Heems Kerk and G. Van der Zwan, Absence of 633-nm laser irradiation-induced effects on glucose phosphorylation by hexokinase, J. Photochem. Photobiol. B 3 (2010), 216-222.

[11] N. Houreld and H. Abrahamselt, Low-intensity laser irradiation stimulates wound healing in diabetic wounded fibroblast cells (WS1), Diabetes Technol. Ther. 12 (2010), 971-979.

[12] R. Lubart, M. Eicher, R. Lavi, H. Friedmann and A. Shainberg, Low-energy laser irradiation promotes cellular redox activity, Photomed. Laser Surg. 23 (2005), 3-9.

[13] R. Lubart, R. Lavi, H. Friedmann and S. Rochkind, Photochemistry and photobiology of light absorption by living cells, Photomed. Laser Surg. 24 (2006), 179-185.

[14] Luxeon Star, SMD red LED data sheet, part number LXML-PD01-0030, available at: http://www.luxeonstar.com/RedLambertian-Rebel-90-lm-p/lxm2-pd01-0040.htm.

[15] C.R.S. Marques, A.A. Martin, C.J. De Lima, L.A.L. Conrado, F.L. Silveira and M.V. Carvalho, The use of hyperbaric oxygen therapy and LED therapy in diabetic foot, Lasers in Surg. XIV, Proc. SPIE, Vol. 5312, 2004, pp. 47-53.

[16] N.C.R. Morais, A.M. Barbosa, M.L. Vale, A.B. Villaverde, C.J. Lima, J.C. Cogo and S.R. Zamuner, Anti-inflammatory effect of low-level laser and light-emitting diode in zymosan-induced arthritis, Photomed. Laser Surg. 28 (2010), $227-232$.

[17] P.C.L. Silveira, L.A. Silva, T.P. Freitas, A. Latini and R.A. Pinho, Effects of low-power laser irradiation (LPLI) at different wavelengths and doses on oxidative stress and fibrogenesis parameters in an animal model of wound healing, Lasers Med. Sci. 26 (2011), 125-131.

[18] A.P.C. Sousa, J.N. Santos, J.A. dos Reis, T.A. Ramos, J. de Souza, M.C.T. Cangussu and A.L.B. Pinheiro, Effect of LED phototherapy of three distinct wavelengths on fibroblasts on wound healing: a histological study in a rodent model, Photomed. Laser Surg. 28 (2010), 547-552. 
[19] SuperBright red LED data sheet, package dimension LED with $5 \mathrm{~mm}$ diameter, part number RL5-R12008, available at: $\mathrm{http} / / /$ www.superbrightleds.com/cgi-bin/store/index.cgi?action=DispPage\&Page2Disp=\%2Fspecs\%2Fr3_specs.htm.

[20] SuperBright red LED data sheet, package dimension with $5 \mathrm{~mm}$, part number RL5-IR2730, available at: http://www. superbrightleds.com/cgi-bin/store/index.cgi?action=DispPage\&Page2Disp=\%2Fspecs\%2FIR2730_specs.htm.

[21] N.G. Yeh, C.H. Wu and C.C. Ta, Light-emitting diodes - their potential in biomedical applications, Ren. Sustain. Energy Rev. 14 (2010), 2161-2166.

[22] G.A. Zalesskayaa and V.S. Ulashchik, Molecular mechanisms of photochemotherapy (review), J. Applied Spectr. 76 (2009), 44-65. 


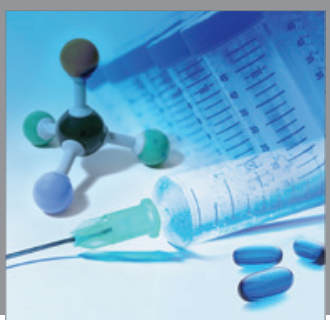

International Journal of

Medicinal Chemistry

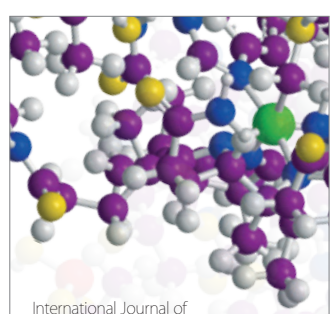

Carbohydrate Chemistry

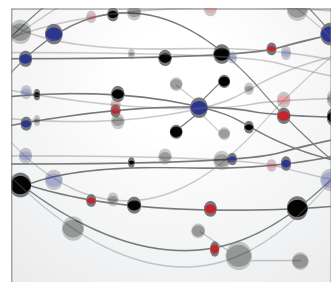

The Scientific World Journal
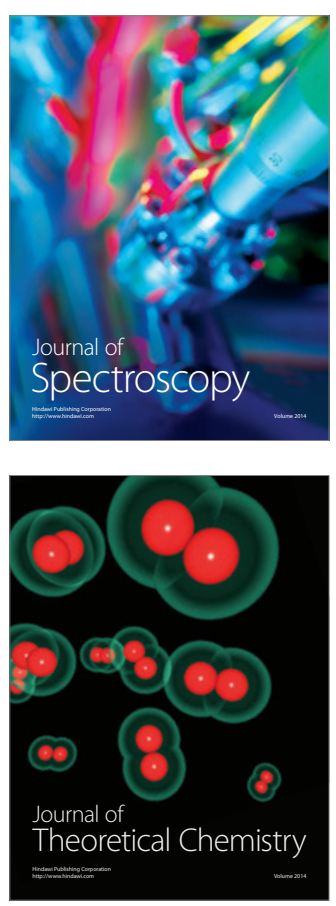
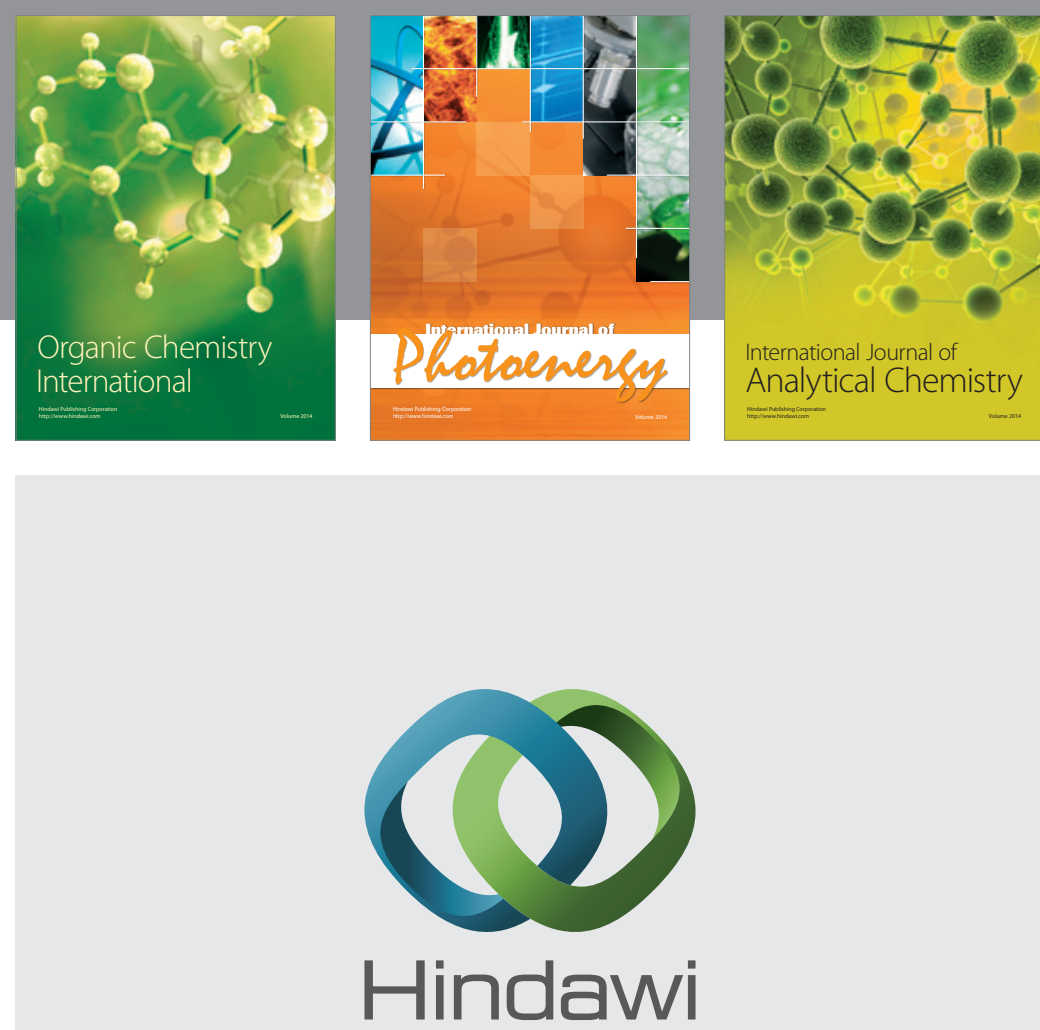

Submit your manuscripts at

http://www.hindawi.com
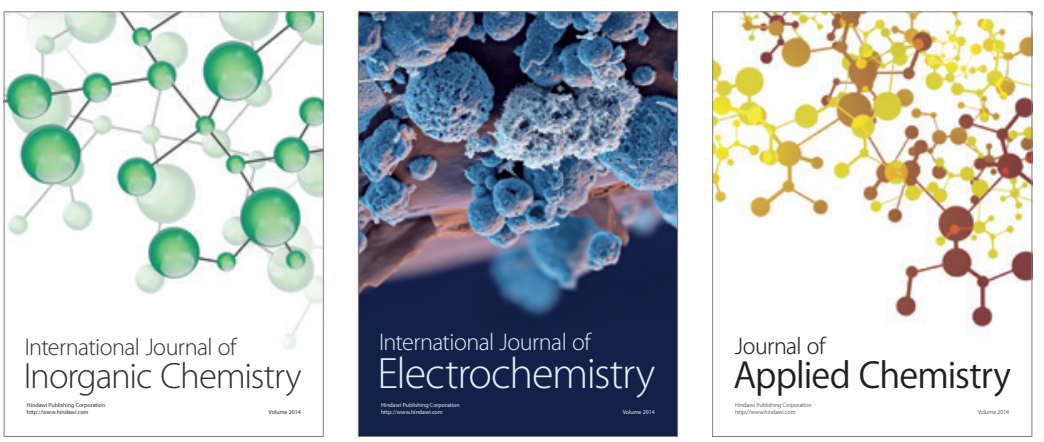

Journal of

Applied Chemistry
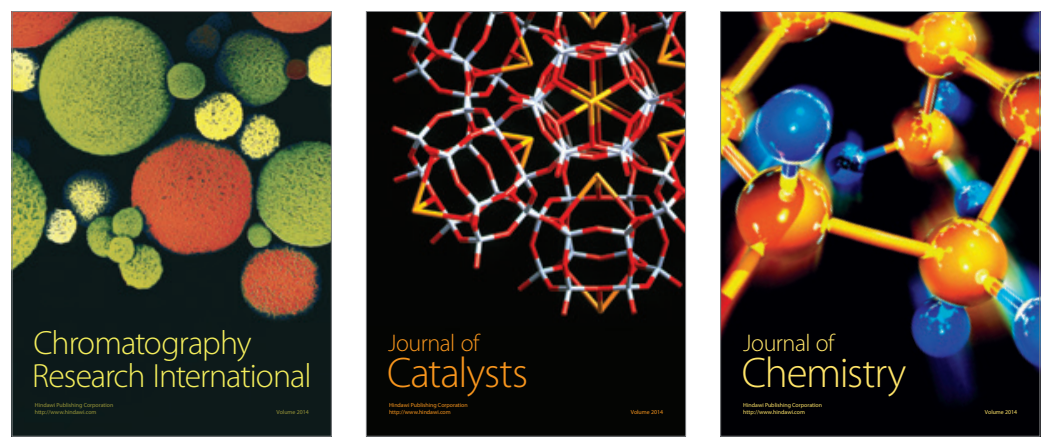
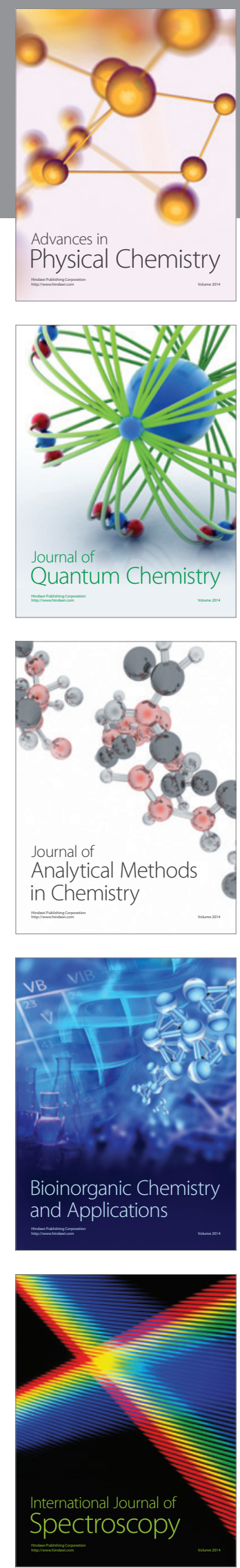